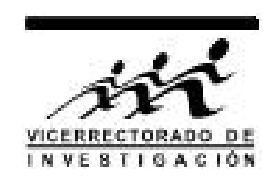

\title{
Determinación del clima de olas en aguas profundas, frente a las costas del Perú mediante calibración de reanálisis numérico e información instrumental
}

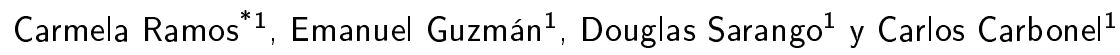 \\ ${ }^{1}$ Universidad Nacional Mayor de San Marcos - Grupo de Investigación GIHREHMA.
}

Recibido 03 julio 2017 - Aceptado 24 setiembre 2017

\begin{abstract}
Para determinar el clima de olas en aguas profundas frente a las costas del Perú, se empleó información instrumental de satélite y boyas, que sirvieron para calibrar los datos de olas del reanálisis numérico del Centro Nacional de Predicción Ambiental (NCEP), que cuenta con información de 1979 a 2017. Las alturas de olas se calibraron mediante la aplicación de un modelo exponencial descrito por Minguez et al. (2011), obteniéndose valores de coeficientes de correlación entre 0.7 a 0.9, el sesgo (BIAS) de -0.03 a 0.01 y el error medio cuadrático (RMSE) calculados mostraron entre 0.17 a 0.33 , lo que indica una buena aproximación entre ambas fuentes de información, por lo que se ha logrado obtener una información de oleaje en aguas profundas que puede ser empleado en análisis de los regímenes medios y extrémales como punto de partida para estudios de ingeniería de costas, así como para estudiar la influencia de los fenómenos El Niño y La Niña sobre el patrón de oleaje frente a la zona de estudio. De esta serie de tiempo calibrada de 38 años, se observó que el oleaje en condiciones medias tiene una altura significante de $2 \mathrm{~m}$, con máximas que pueden superar los $4 \mathrm{~m}$. La dirección predominante de olas es de dirección Suroeste para las zonas norte y centro y dirección Sursuroeste para la zona sur. Asimismo, se observó que durante la ocurrencia de los fenómenos EI NIÑO y La NIÑA, se presentó un incremento de las alturas significante de olas en todos los puntos evaluados. La dirección de olas durante un evento La Niña, mostró dirección predominante del Sursuroeste, mientras que en fenómeno El Niño se observó dirección predominante del Suroeste.
\end{abstract}

Palabras claves: Clima de olas, calibración de olas Perú.

\section{Determination of waves climate in deep waters, offshore Peru through calibration of numerical reanalysis and instrumental information}

To determinate the wave climate in deep water off the Peruvian coast, instrumental data from satellite and buoys was employed to calibrate wave reanalysis from NCEP for the period 1979 to 2017. Wave data was calibrated using an exponential model described by Minguez et al. (2011). The results shows correlation coefficient values between 0.7 to 0.9 , also BIAS and RMSE showed acceptable values, which indicate a good approximation between both information databases. We have obtained wave information in deep waters which can be used to analyze average an extremely wave regimes to study coastal processes, and study the influence of EI NIÑO and La NIÑA phenomenon in the study area. This calibrated wave time series reveals that the average conditions for wave height is around $2 \mathrm{~m}$, with maximum heights over 4 $\mathrm{m}$. De predominant direction is from Southest for the northern and center zones, and South-southwest for southern zone. In other hand, it was observed that during EI NIÑO and La NIÑA wave height was incremented in all the control points selected. The wave direction during La NIÑA showed predominant direction from South-southwest, while during EI NIÑO was observed predominant direction from Southwest. Keywords: wave climate, wave data calibration, Peru.

\section{Introducción}

Las condiciones de oleaje que se presentan a nivel costero dependen en gran medida de las características de las olas en aguas profundas, principalmente de aquellas olas que salen de las zonas de generación. Es conocido que en el océano Pacífico se presentan dos zonas de generación de oleaje, una en cada hemisferio. El Perú se encuentra

*carmela.ramoso@ciplima.org.pe 
ubicado en la parte occidental del Océano Pacífico y recibe las olas que provienen principalmente de la zona de generación del hemisferio Sur, ubicada aproximadamente en $40^{\circ}$ latitud Sur (Ge Chen et al., 2002).

Los estudios a nivel costero relacionados al clima marítimo y la dinámica de litoral requieren del conocimiento detallado de las condiciones de oleaje en aguas profundas, por lo que se requiere de datos por periodos largos de tiempo, que permita establecer las condiciones medias y extremas, así como las variaciones estacionales. En este sentido las mediciones con boyas ofrecen registros temporales precisos, pero tienen la limitación que presentan periodos cortos y muy distanciados en el tiempo y por lo general están ubicadas en el hemisferio norte (Reguero et al., 2012). Asimismo, la información satelital ofrece cobertura global de oleaje de buena precisión, con una variación de $\pm 3 \mathrm{~cm}$ (Krogstad \& Barstow, 1999), por lo que la hace una buena fuente de información, la deficiencia es que no se cuenta con información de mediciones de dirección de las olas. En este sentido, en las últimas décadas se ha hecho necesario el empleo de modelos numéricos de oleaje a nivel global para la generación de información de olas por periodos largos; sin embargo, esta información en términos cuantitativos no es totalmente perfecta (Reguero et al., 2012), por lo que necesita un tratamiento adecuado de los datos y calibrado.

Por otro lado, los fenómenos El Niño y La Niña afectan a las condiciones ambientales, sobre todo del océano Pacifico central, afectando las costas del Perú. En la actualidad la mayor parte de estudios del océano respecto a estos fenómenos, se basan en investigar, las variaciones de temperaturas superficiales del mar (B.Dewitte et al., 2011), salinidad (Mei Zhao et al., 2016; Gregory C. Johnson et al.,2002), nivel medio del mar (Klaus Wyrtki, 1977) o las precipitaciones (Lavado-Casimiro \& Espinoza, 2014), siendo estos parámetros los más importantes para evaluaciones de El Niño y La Niña, poniéndose más énfasis en determinar los incrementos que se dan en el océano debido a las variaciones que esto ocasiona en zonas costeras.

Asimismo, los principales estudios realizados sobre la dinámica de olas, se basan en el Hemisferio Norte, debido a la presencia de Huracanes o ciclones (Macafee \& Wong, 2007), quedando de lado, el análisis de una climatología de olas en el Hemisferio Sur que permita evaluar evidencias de la presencia de EI Niño o La Niña, la cual, a través de los años, ha sido estudiada de forma regional o como país, dejando de lado el análisis de un solo sistema oceánico. De esta manera, entre los pocos estudios que se han realizado en Océano Pacifico con énfasis en el hemisferio Sur, tenemos a nivel regional (Scott A. Stephens \& D.L. Ramsay, 2014) , a nivel global (Ge Chen et al., 2002, Reguero et al.,2012), y como países tenemos los atlas de clima de olas en Colombia (Francois-Thomas et al., 2016), Ecuador (Vera San Martín \& Marín Villalta, 2015), Chile (Beyá M. et al., 2016) por lo que este estudio contribuiría a crear una base para caracterizar las olas para el Perú. También existen estudios que evidencian que el cambio climático también puede ser evaluado mediante el análisis de la variabilidad a largo plazo de las olas (Mark A. Hemer et al., 2013; Hemer et al., 2010). Vera \& Marin (2015) determinaron el clima de olas en el Océano Pacífico mediante información satelital y compararon las características de las olas en años normales con la ocurrencia de fenómeno EI Niño y La Niña, donde se concluye que existe un incremento en las alturas de olas sobre todo en ocurrencia de Niños fuertes o moderados.

De lo anteriormente descrito, el objetivo del presente estudio es realizar la calibración de los datos de reanálisis numérico del Centro Nacional de Predicción Ambiental (NCEP, por sus siglas en Ingles.) con información instrumental de satélite y boyas disponibles para la región Perú y áreas adyacentes, con la finalidad de establecer el clima de olas (base) y analizar la influencia de eventos extremos como son los fenómenos El Niño y La Niña sobre las características del oleaje frente a las costas del Perú y áreas adyacentes.

\section{Materiales y Métodos}

Para la realización de esta investigación se ha empleado información instrumental de oleaje en aguas profundas, tales como la información de la boya 32012 del National Oceanic and Atmospheric Administration (NOAA), que se encuentra ubicada dentro de la zona de estudio proyectada en esta investigación, e información de satélite que permitirá calibrar los datos de reanálisis numérico proveniente del modelo Wave Watch (WWATCH) del NCEP (Tolman, H.L. et al.,2002; Chawla et al., 2013).

La boya 32012 (NOAA, National Data Buoy Center, 2017) se encuentra ubicada en las coordenadas $19.425^{\circ}$ Sur y $85.078^{\circ}$ Oeste, donde se dispone de información desde el año 2007 al 2016 (Tabla 1), de altura significante, dirección y periodo pico de oleaje. Complementando a esta información, se tiene información proveniente del proyecto Globwave (GlobWave, 2013), que cuenta con datos de altimetría de los satélites GEOSAT, ENVISAT, JASON 1, ERS1, ERS2 y TOPEX/POSEIDON (Tabla 1). Se seleccionaron 23 puntos (Figura 1, derecha) como representativos para ser empleados en la calibración. Cada uno de los 23 puntos seleccionados tiene un radio de acción aproximado de $1^{\circ}$ en aguas profundas y un radio aproximado de $0.5^{\circ}$ para los puntos ubicados más cerca a costa donde se extrae la información de satélite con la finalidad de obtener series temporales de oleaje en cada punto.

La información de reanálisis numérico fue obtenida del NCEP - NOAA, seleccionándose los datos que corresponden al área de estudio (Figura 1, derecha). En esta base de datos se dispone dos periodos de información, la primera 

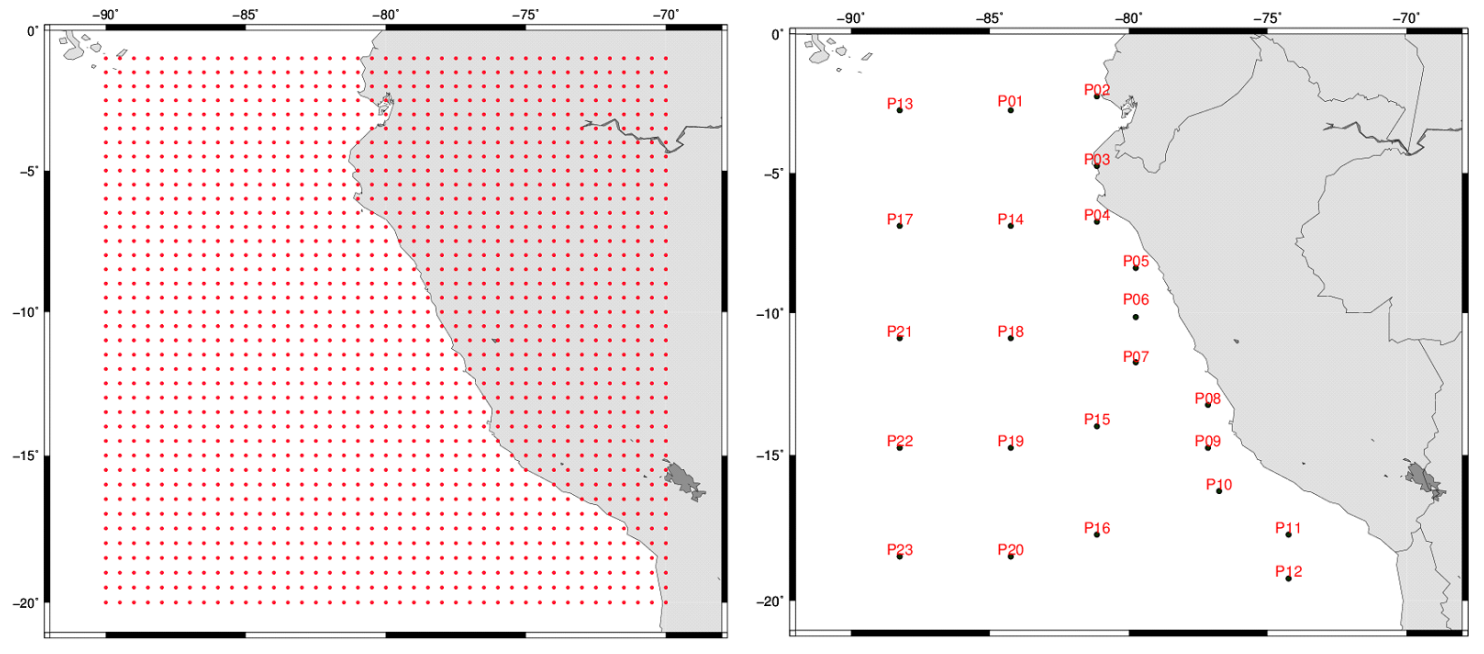

Figura 1: Área de Estudio. Derecha: Puntos de grilla del modelo Wave Watch III. Izquierda: Puntos de selección de datos de satélite. Fuente: Elaboración propia

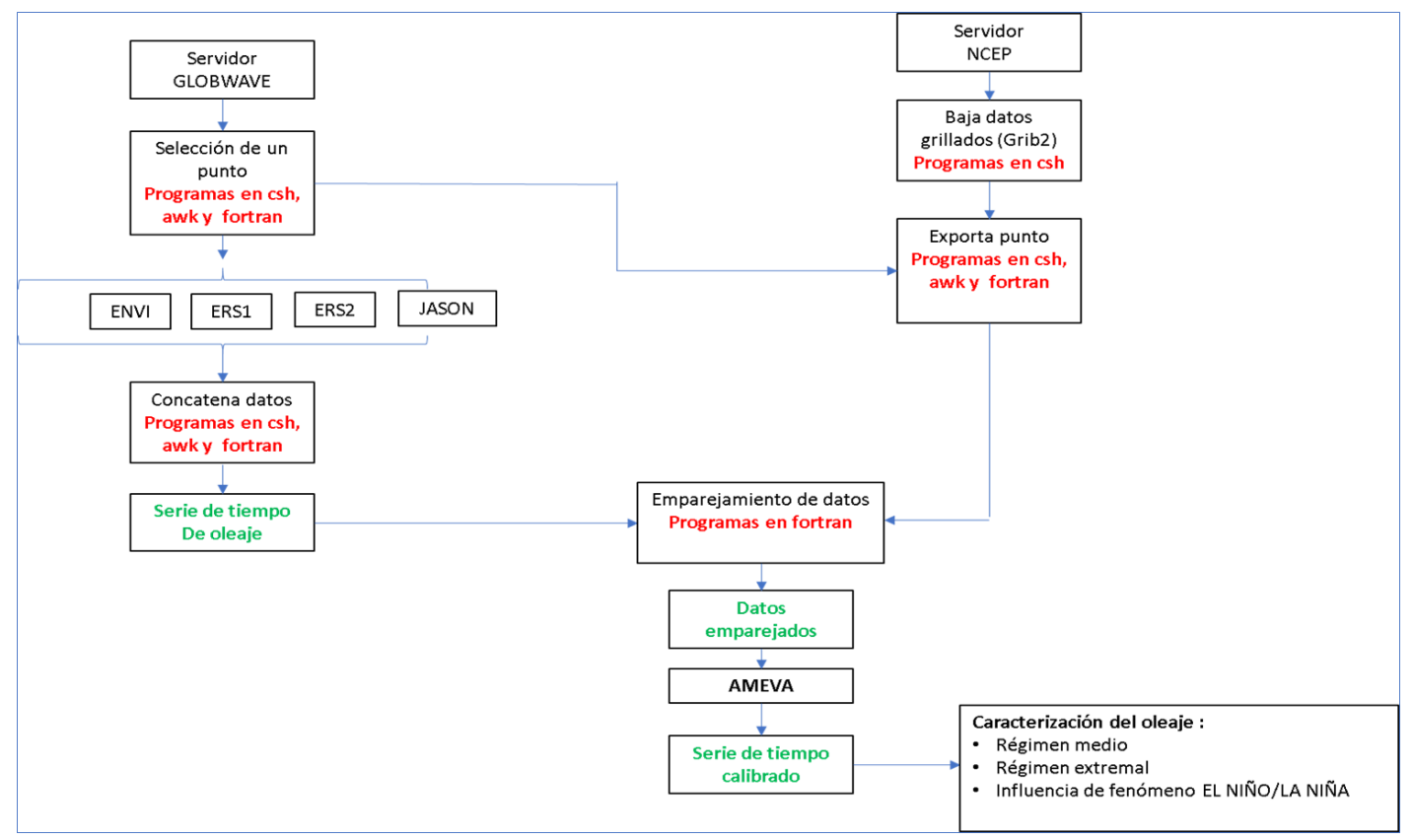

Figura 2: Esquema general del proceso de calibración de los datos de oleaje. Fuente: elaboración propia. 


\begin{tabular}{|c|c|c|}
\hline Información & Descripción & Periodo de Información \\
\hline Boya & 32012 & $2007-2016$ \\
\hline \hline \multirow{3}{*}{ Satélite } & Envisat(ENVI) & $2002-2012$ \\
\cline { 2 - 3 } & ERS-1(ERS1) & $1991-1996$ \\
\cline { 2 - 3 } & ERS-2(ERS2) & $1995-2009$ \\
\cline { 2 - 3 } & Geosat(GEOS) & $1985-1989$ \\
\cline { 2 - 3 } & Jason1(JAS1) & $2002-2010$ \\
\cline { 2 - 3 } & Topex/Poseidon(TOPX) & $1992-2005$ \\
\hline
\end{tabular}

Tabla 1: Información empleada en el proceso de calibración. Fuente: (Farquhar et al, 2013)

\begin{tabular}{|c|c|c|c|c|c|}
\hline \multicolumn{2}{|c|}{ Inicio } & \multicolumn{2}{c|}{ Final } & \multirow{2}{*}{ Duración } & \multirow{2}{*}{ Magnitud } \\
\hline Año & Mes & Año & Mes & \multicolumn{2}{|c|}{} \\
\hline 1951 & 5 & 1951 & 10 & 6 & Moderado \\
\hline 1953 & 3 & 1953 & 6 & 4 & Débil \\
\hline 1957 & 3 & 1958 & 5 & 15 & Moderado \\
\hline 1965 & 3 & 1965 & 10 & 8 & Moderado \\
\hline 1969 & 4 & 1969 & 7 & 4 & Moderado \\
\hline 1972 & 3 & 1973 & 2 & 12 & Fuerte \\
\hline 1976 & 5 & 1976 & 10 & 6 & Débil \\
\hline 1982 & 7 & 1983 & 11 & 17 & Extraordinario \\
\hline 1986 & 12 & 1987 & 12 & 13 & Moderado \\
\hline 1991 & 10 & 1992 & 6 & 9 & Moderado \\
\hline 1993 & 3 & 1993 & 9 & 7 & Débil \\
\hline 1994 & 11 & 1995 & 1 & 3 & Débil \\
\hline 1997 & 3 & 1998 & 9 & 19 & Extraordinario \\
\hline 2002 & 3 & 2002 & 5 & 3 & Débil \\
\hline 2002 & 9 & 2003 & 1 & 5 & Débil \\
\hline 2003 & 11 & 2004 & 1 & 3 & Débil \\
\hline 2004 & 10 & 2004 & 12 & 3 & Débil \\
\hline 2006 & 8 & 2007 & 2 & 7 & Moderado \\
\hline 2008 & 7 & 2008 & 9 & 3 & Débil \\
\hline 2009 & 5 & 2009 & 10 & 6 & Débil \\
\hline
\end{tabular}

Tabla 2: Ocurrencia de El Niño en la Costa del Perú. Fuente: (ENFEN, 2012) 
de ellas corresponde al periodo de 1979 al 2009 (NOAA, Marine Modeling and Analysis Branch) y la segunda al periodo de febrero 2005 a julio 2017 (Environmental Modeling (enter). Ambas fuentes fueron concatenadas para lograr obtener un periodo de información de olas desde 1979 hasta julio 2017, contabilizando un total de 38 años. Con la finalidad de estudiar el comportamiento del oleaje durante la ocurrencia de eventos extremos como El Niño - La Niña se ha tomado como referencia la clasificación de años dada por las instituciones conformantes del ENFEN. De esta manera se tiene que los años 1982-1983 y 1997-1998 son considerados como Niños extraordinarios y los años 1975, 1988 y 2007 como La Niña Fuerte (CENEPRED, 2012) (ENFEN, 2012).

\section{Metodología}

El área seleccionada para esta investigación es presentada en la Figura 1 y comprende un área aproximada de $2300 \mathrm{~km} \times 2300 \mathrm{~km}$, donde se han definido 23 puntos con el objetivo de comparar la información de reanálisis numérico con la información instrumental, y de esta forma calibrar la información del modelo. Un esquema general del proceso de calibración se muestra en la Figura 2 y se describe a continuación:

- Se implementaron programas en csh, awk y fortran, los cuales permitieron extraer los datos del servidor Globwave de forma operacional para cada satélite y realizar un concatenado de datos para finalmente obtener una serie de tiempo de altura de olas que corresponde a cada punto seleccionado y mostrado en la Figura 1-derecha. Un ejemplo de la ruta de satélite se muestra en la Figura 3.

- Se extrajo los datos del reanálisis de olas del servidor del NCEP, implementándose programas en csh, awk y fortran para obtener puntos de olas de 1979 a 2017 y mostrados en la Figura 1-izquierda.

- Una vez que se ha obtenido la información de satélite y del reanálisis numérico (para el punto que coincide con el satélite), se procede con el proceso de emparejamiento de información, es decir se selecciona la fecha donde ambas fuentes de información coincidan y un ejemplo se muestra en la Figura 4.

- En la calibración del modelo se empleó el software AMEVA desarrollado por el Instituto de Hidráulica Ambiental de la Universidad de Cantabria (IHCantabria, 2014), el que permitió determinar los coeficientes de calibración ("a" y "b") para las alturas de olas, por cada dirección de oleaje; para los 23 puntos seleccionados (Figura 1-derecha), este proceso de calibración es sugerido por Minguez (2011).
- Con los 23 puntos de olas calibrados en altura de olas, se procede a calibrar los otros puntos de olas provenientes del NCEP, empleando los coeficientes de calibración calculados anteriormente denominados a y b, los mismos que se describirán más adelante. Obteniéndose de esta manera una grilla de $41 \times 41$ puntos de olas calibradas, con un espaciado cada $0.5^{\circ}$ (Figura 1-izquierda).

- Para el procesamiento de información y evaluación del fenómeno El Niño y Niña en las olas, se emplearon los datos de alturas de olas calibradas, implementándose programas en el software $R$ y para la elaboración de planos espaciales se empleó el Generic Mapping Tools (GMT).

Todos estos pasos de calibración del modelo se repiten para el punto de la boya 32012 ubicada en $19.425^{\circ}$ Sur $85.078^{\circ}$ Oeste y un punto adicional de olas proveniente del reanálisis del NCEP para la misma ubicación.

\section{Proceso de calibración}

Lo descrito anteriormente, muestra un sistema completo que permite realizar desde la descarga de datos hasta la calibración del punto de oleaje seleccionado (Figura 2). El proceso de calibración sugerido por Minguez (2011), donde la altura de ola calibrada se calcula mediante la aplicación de un modelo exponencial de la siguiente manera:

$$
H_{s}^{c}=a(\theta)\left(H_{s}^{R}\right)^{b(\theta)}
$$

Donde:

$H_{s}^{c}$ : Altura de ola de reanálisis numérico calibrada.

$H_{s}^{R}$ : Altura de ola de reanálisis numérico sin calibrar.

$a(\theta)$ y $b(\theta)$ : son los coeficientes de calibración que van a ser determinados por cada dirección de aproximación de oleaje.

Una vez que se han obtenido los coeficientes "a" y "b", se procede a aplicar el modelo exponencial a toda la serie de tiempo (1979 a 2017), obteniéndose de esta manera todos los datos de oleaje calibrados por cada dirección de olas en cada punto de grilla.

\section{Resultados}

Con la información descargada, se realizó una comparación entre los resultados del reanálisis numérico con la información satelital para cada punto definido. Se observa que la información del reanálisis numérico sin calibrar con los datos instrumentales presenta una correlación alta, presentando un coeficiente de correlación que varía entre 0.727 y 0.933 (Tabla 4), asimismo, observamos que los 


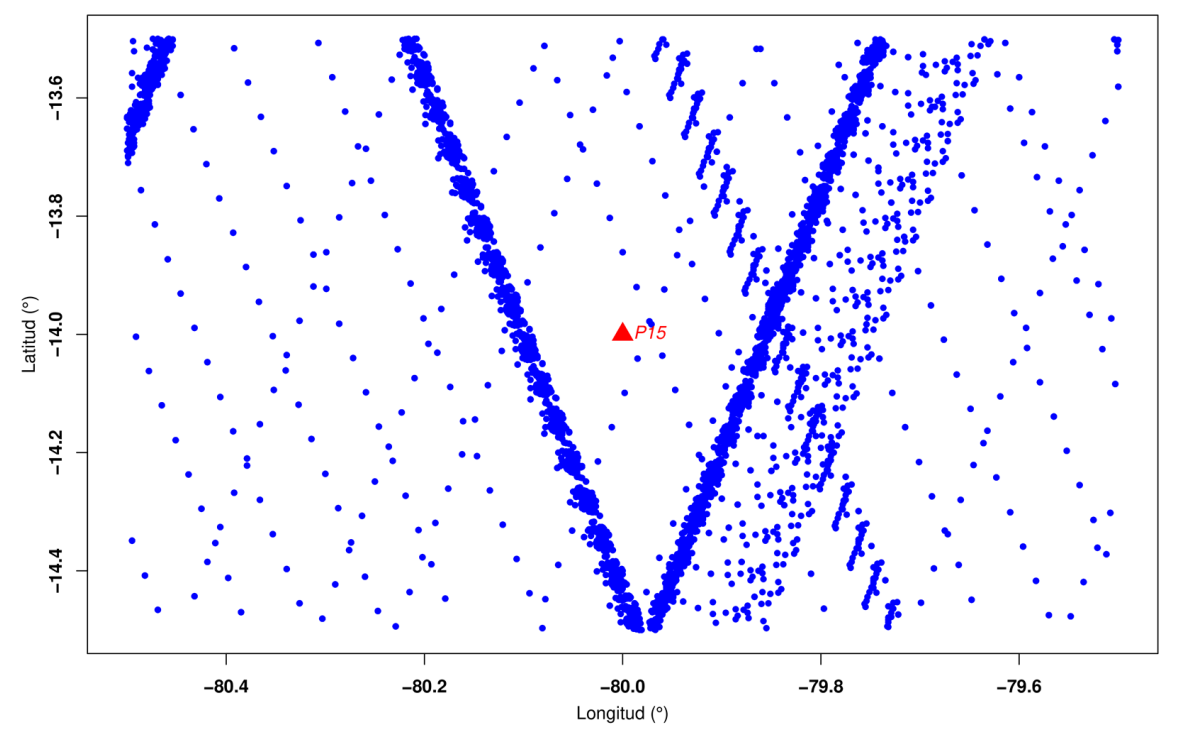

Figura 3: Ruta de los satélite (GEOSAT, ENVISAT, JASON 1, ERS1, ERS2 y TOPEX/POSEIDON) empleados para el punto P15. Fuente: elaboración propia.

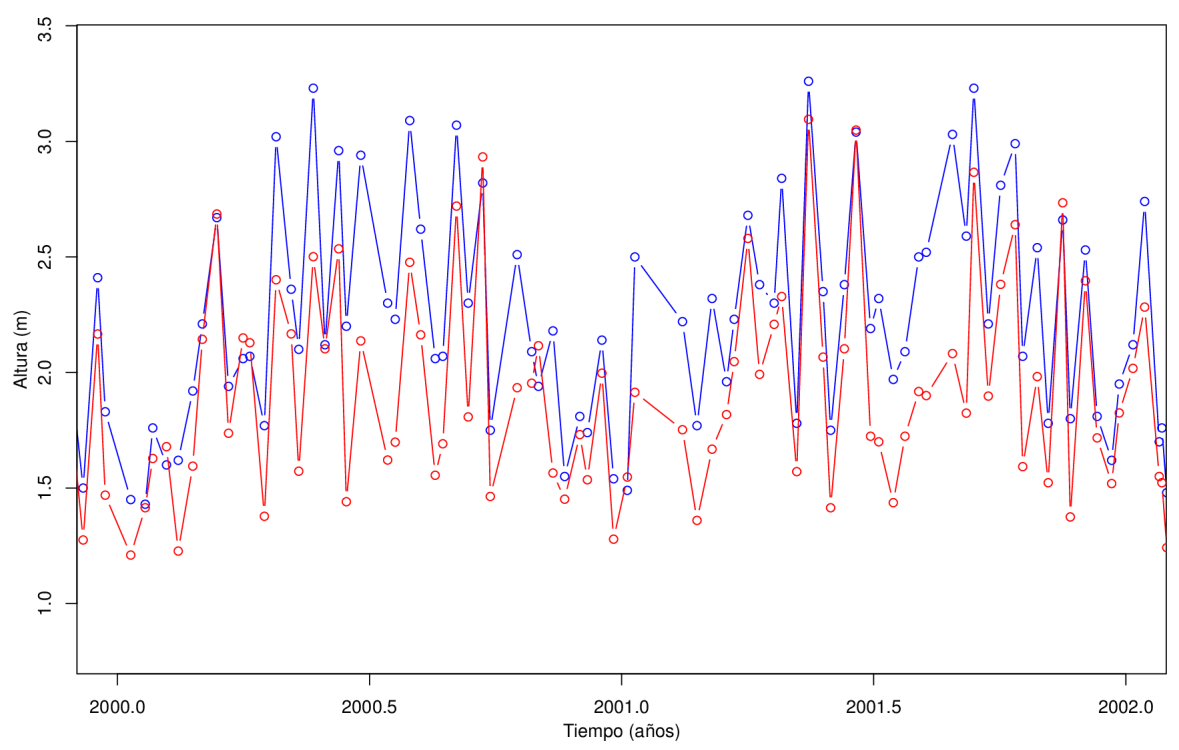

Figura 4: Emparejado de altura de olas entre el modelo y satélite para el punto P07. En azul los datos del modelo y rojo el satélite. 
valores del BIAS mantienen rangos entre 0.259 a 0.427 y el RMSE entre 0.337 a 0.532 . Cuando se realiza la calibración, se obtienen los coeficientes de calibración "a" y "b" que permiten aproximar la información de reanálisis a un modelo exponencial descrito en la sección anterior. De esta manera se observa que los coeficientes de correlación ( $r$ ) mantienen valores muy similares; sin embargo, los valores del BIAS y RMSE se ven reducidos considerablemente, lo que nos daría indicios de que el proceso de calibración mejoró la información de reanálisis numérico. Por otro lado, el p-value para el coeficiente de correlación de ambas fuentes de información indica que la correlación es significante (Tabla 4).

El proceso de calibración de la boya con los datos del modelo, muestran una mejora tanto en la correlación como en los BIAS y RMSE. En la figura 5 y 6 se observa el gráfico de dispersión entre la información de reanálisis sin calibrar y calibradas con la información instrumental de satélite y la boya 32012 respectivamente.

Con los coeficientes "a" y "b" obtenidos en cada punto, se procede a aplicar dicha aproximación a la totalidad de puntos extraídos, de manera que se obtuvo la distribución espacial para los cuantiles de $50 \%, 95 \%$ y máxima (Figura 7), observándose que la altura significante presenta mayores valores hacia la dirección suroeste en aguas profundas. Asimismo, de manera general se observó que las mayores alturas de olas se presentan en la zona sur y van disminuyendo hacia la zona norte.

La dirección de olas (Figura 8), es predominantemente de dirección Sur-Suroeste y conforme se va aproximando a la zona costera se producen pequeños cambios de dirección hacia el Suroeste para el cuantil de $50 \%$. En el cuantil de $95 \%$ observamos eventos en los que el oleaje viene de dirección Oeste y conforme se acerca a costa se modifica y cambia a dirección Suroeste. En eventos máximos, se puede observar presencia de oleaje del Noroeste. La distribución espacial del periodo de olas (Figura 9) muestra que al cuantil de $50 \%$ el periodo de olas predominante es entre 14 a 15 segundos. Para el cuantil 95\% se muestra periodos entre 17.5 a 18.5 segundos, mientras que, para eventos máximos, se evidencia periodos entre 23 a 24 segundos.

\section{Caracterización de olas en la zona norte, centro y sur} del país

Para complementar lo descrito en los párrafos anteriores, se han seleccionado tres puntos de control (Figura 1 ), donde se representará las características de las olas en la zona norte, centro y sur del país, las mismas que se describen a continuación:

- Zona Norte del país (Punto P02).- Longitud: $82^{\circ}$ Oeste y Latitud: $03^{\circ}$ Sur

- Zona Centro del país (Punto P07). - Longitud: $79^{\circ}$ Oeste y Latitud: $11.5^{\circ} \mathrm{Sur}$
- Zona Sur del país (Punto P11). - Longitud: $76^{\circ}$ Oeste y Latitud: $18^{\circ}$ Sur

En cada una de las ubicaciones puntuales, se tiene información de 38 años de olas calibradas con información instrumental (satélite y boya), empleándose estos datos para construir una rosa de altura de ola y periodo de ola para cada punto, mostrando la variabilidad direccional existente en el oleaje. Observamos de manera general que el oleaje presenta dirección predominante del Sur-Suroeste en la zona norte y centro del Perú, mientras que la zona sur del Perú, la dirección predominante es Suroeste.

En la zona norte del país (P02), las olas tienen una dirección Sur-suroeste, con alturas de olas predominantes entre 1.5 a $2.0 \mathrm{~m}$, y periodos entre 14 a 16 segundos, sin embargo, se observa que en oleaje del Noroeste las alturas de olas entre 1.0 a $1.5 \mathrm{~m}$ con periodos entre 16 a 18 segundos.

En la zona centro (P07), las olas tienen una dirección Sur-suroeste, mostrándose que las alturas de olas se ven intensificadas respecto a la zona norte, con alturas entre 1.5 a $2.5 \mathrm{~m}$ y periodos entre 12 a 16 segundos, así mismo, las olas de dirección Noroeste y Oeste-Noroeste presentan alturas entre 1.0 a $1.5 \mathrm{~m}$ y periodos entre 16 a 18 segundos predominantemente.

El punto ubicado en la zona sur del Perú (P11), muestra que la dirección predominante para esa zona es de dirección Suroeste con alturas predominantes entre 1.5 a $2.5 \mathrm{~m}$ con periodos entre 12 a 16 segundos y las olas de dirección Noroeste y Oeste-Noroeste presentan alturas entre 1.5 a $2.0 \mathrm{~m}$ y periodos entre 16 a 18 segundos predominantemente.

\section{Régimen Medio de oleaje}

El régimen medio de oleaje está referido al conjunto de estados de oleaje más probable que podemos encontrar (estado) de una serie de temporal. La Tabla 5 muestra que el régimen medio esta entre 1.62 y $2.19 \mathrm{~m}$ al $50 \%$ de cuantiles de altura de ola, mientras que para el periodo 13.7 a 14.3 segundos.

\section{Régimen extremal de oleaje}

Las distribuciones de régimen extremal denominadas Generalized Extreme Value distribution (GEV) y Gumbel, se muestran para los 3 puntos de olas elegidos (P02, P07 y P11), en los que se calculó un periodo de retorno de ola de hasta 50 años, en la que se observa lo siguiente:

- La zona norte (P02) muestra altura de olas entre 3.10 a $3.15 \mathrm{~m}$ para un periodo de retorno de 2 años, mientras que para 10 años las alturas son de 3.49 a $3.66 \mathrm{~m}$, amplificándose hasta 3.62 a $4.16 \mathrm{~m}$ en 50 años.

- El punto P07 correspondiente a la zona centro del Perú, muestra para 2 años de periodo de retorno, 


\begin{tabular}{|c|c|c|c|c|c|}
\hline \multicolumn{2}{|c|}{ Inicio } & \multicolumn{2}{c|}{ Final } & \multirow{2}{*}{ Duración } & \multirow{2}{*}{ Magnitud } \\
\hline Año & Mes & Año & Mes & & \\
\hline 1950 & 2 & 1950 & 12 & 11 & Fuerte \\
\hline 1952 & 8 & 1952 & 10 & 3 & Débil \\
\hline 1954 & 1 & 1956 & 2 & 26 & Fuerte \\
\hline 1956 & 9 & 1956 & 12 & 4 & Moderado \\
\hline 1960 & 5 & 1960 & 7 & 3 & Débil \\
\hline 1961 & 6 & 1961 & 10 & 5 & Débil \\
\hline 1962 & 2 & 1962 & 8 & 7 & Fuerte \\
\hline 1964 & 3 & 1964 & 11 & 9 & Fuerte \\
\hline 1966 & 4 & 1966 & 7 & 4 & Moderado \\
\hline 1967 & 7 & 1968 & 6 & 12 & Fuerte \\
\hline 1970 & 4 & 1971 & 11 & 20 & Fuerte \\
\hline 1973 & 5 & 1974 & 2 & 10 & Moderado \\
\hline 1974 & 10 & 1975 & 1 & 4 & Moderado \\
\hline 1975 & 7 & 1976 & 1 & 7 & Fuerte \\
\hline 1978 & 4 & 1978 & 9 & 6 & Débil \\
\hline 1985 & 2 & 1985 & 9 & 8 & Moderado \\
\hline 1988 & 5 & 1988 & 10 & 6 & Fuerte \\
\hline 1996 & 4 & 1996 & 7 & 4 & Débil \\
\hline 2001 & 9 & 2001 & 12 & 4 & Débil \\
\hline 2007 & 5 & 2007 & 12 & 8 & Fuerte \\
\hline 2010 & 8 & 2010 & 11 & 4 & Moderado \\
\hline
\end{tabular}

Tabla 3: Ocurrencia de La Niña en la Costa del Perú. Fuente: (ENFEN, 2012)

\begin{tabular}{|c|c|c|c|c|c|c|c|c|c|c|}
\hline \multirow{2}{*}{ Punto } & \multirow{2}{*}{ Longitud } & \multirow{2}{*}{ Latitud } & \multirow{2}{*}{$\mathrm{n}$} & \multicolumn{3}{|c|}{ Sin Calibrar } & \multicolumn{3}{|c|}{ Calibrado } & \multirow{2}{*}{$\mathrm{p}$-value } \\
\hline & & & & $r$ & BIAS & RMSE & $r$ & BIAS & RMSE & \\
\hline P01 & -84.0 & -2.0 & 436 & 0.837 & 0.319 & 0.389 & 0.842 & -0.006 & 0.209 & $<2.2 \mathrm{E}-16$ \\
\hline P02 & -82.0 & -3.0 & 858 & 0.823 & 0.268 & 0.345 & 0.821 & -0.004 & 0.209 & $<2.2 \mathrm{E}-16$ \\
\hline P03 & -82.5 & -5.0 & 519 & 0.874 & 0.331 & 0.392 & 0.876 & 0.005 & 0.189 & $<2.2 \mathrm{E}-16$ \\
\hline P04 & -82.0 & -7.0 & 677 & 0.807 & 0.305 & 0.420 & 0.808 & -0.002 & 0.267 & $<2.2 \mathrm{E}-16$ \\
\hline P05 & -80.5 & -8.5 & 886 & 0.846 & 0.376 & 0.443 & 0.846 & -0.006 & 0.215 & $<2.2 \mathrm{E}-16$ \\
\hline P06 & -80.0 & -10.0 & 924 & 0.877 & 0.317 & 0.390 & 0.877 & -0.025 & 0.209 & $<2.2 \mathrm{E}-16$ \\
\hline P07 & -79.0 & -11.5 & 817 & 0.859 & 0.359 & 0.438 & 0.858 & -0.015 & 0.231 & $<2.2 \mathrm{E}-16$ \\
\hline P08 & -78.0 & -13.0 & 531 & 0.829 & 0.360 & 0.456 & 0.830 & -0.015 & 0.251 & $<2.2 \mathrm{E}-16$ \\
\hline P09 & -78.0 & -15.0 & 724 & 0.894 & 0.355 & 0.423 & 0.894 & -0.017 & 0.212 & $<2.2 \mathrm{E}-16$ \\
\hline $\mathrm{P} 10$ & -76.0 & -16.5 & 427 & 0.777 & 0.314 & 0.437 & 0.777 & -0.013 & 0.286 & $<2.2 \mathrm{E}-16$ \\
\hline P11 & -76.0 & -18.0 & 715 & 0.884 & 0.372 & 0.448 & 0.884 & -0.017 & 0.227 & $<2.2 \mathrm{E}-16$ \\
\hline P12 & -73.0 & -19.0 & 544 & 0.727 & 0.406 & 0.532 & 0.729 & -0.028 & 0.327 & $<2.2 \mathrm{E}-16$ \\
\hline P13 & -88.0 & -2.0 & 303 & 0.859 & 0.259 & 0.337 & 0.863 & -0.004 & 0.200 & $<2.2 \mathrm{E}-16$ \\
\hline P14 & -84.0 & -6.0 & 431 & 0.902 & 0.293 & 0.357 & 0.905 & -0.004 & 0.179 & $<2.2 \mathrm{E}-16$ \\
\hline P15 & -80.0 & -14.0 & 699 & 0.865 & 0.357 & 0.438 & 0.865 & -0.004 & 0.231 & $<2.2 \mathrm{E}-16$ \\
\hline P16 & -80.0 & -18.0 & 516 & 0.870 & 0.425 & 0.506 & 0.870 & 0.000 & 0.252 & $<2.2 \mathrm{E}-16$ \\
\hline P17 & -88.0 & -6.0 & 388 & 0.834 & 0.296 & 0.399 & 0.838 & 0.004 & 0.250 & $<2.2 \mathrm{E}-16$ \\
\hline P18 & -84.0 & -10.0 & 436 & 0.813 & 0.375 & 0.482 & 0.810 & -0.004 & 0.266 & $<2.2 \mathrm{E}-16$ \\
\hline P19 & -84.0 & -14.0 & 700 & 0.907 & 0.417 & 0.483 & 0.908 & -0.008 & 0.213 & $<2.2 \mathrm{E}-16$ \\
\hline P20 & -84.0 & -18.0 & 567 & 0.914 & 0.421 & 0.485 & 0.914 & -0.012 & 0.213 & $<2.2 \mathrm{E}-16$ \\
\hline P21 & -88.0 & -10.0 & 514 & 0.933 & 0.406 & 0.449 & 0.933 & 0.008 & 0.169 & $<2.2 \mathrm{E}-16$ \\
\hline P22 & -88.0 & -14.0 & 242 & 0.933 & 0.427 & 0.475 & 0.931 & -0.011 & 0.182 & $<2.2 \mathrm{E}-16$ \\
\hline P23 & -88.0 & -18.0 & 260 & 0.924 & 0.406 & 0.459 & 0.925 & -0.006 & 0.188 & $<2.2 \mathrm{E}-16$ \\
\hline Boya 32012 & -85.078 & -19.425 & 21269 & 0.886 & 0.556 & 0.631 & 0.887 & -0.017 & 0.282 & $<2.2 \mathrm{E}-16$ \\
\hline
\end{tabular}

Tabla 4: Estadística de la información de alturas de olas sin calibrar y calibrado comparado con información satelital. Fuente: propia 

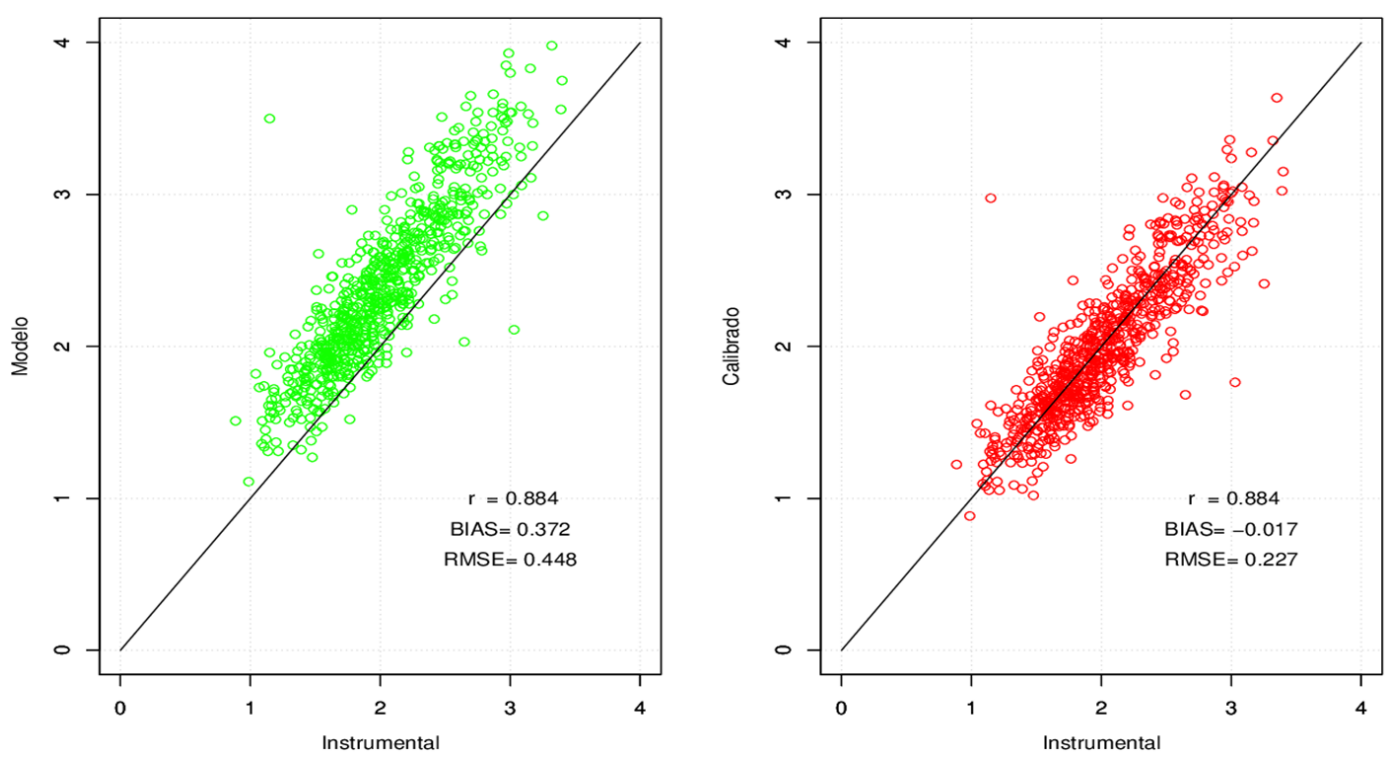

Figura 5: Correlación entre la información de reanálisis e instrumental, punto P07. Izquierda: sin calibrar. Derecha: calibrado. Fuente: propia.
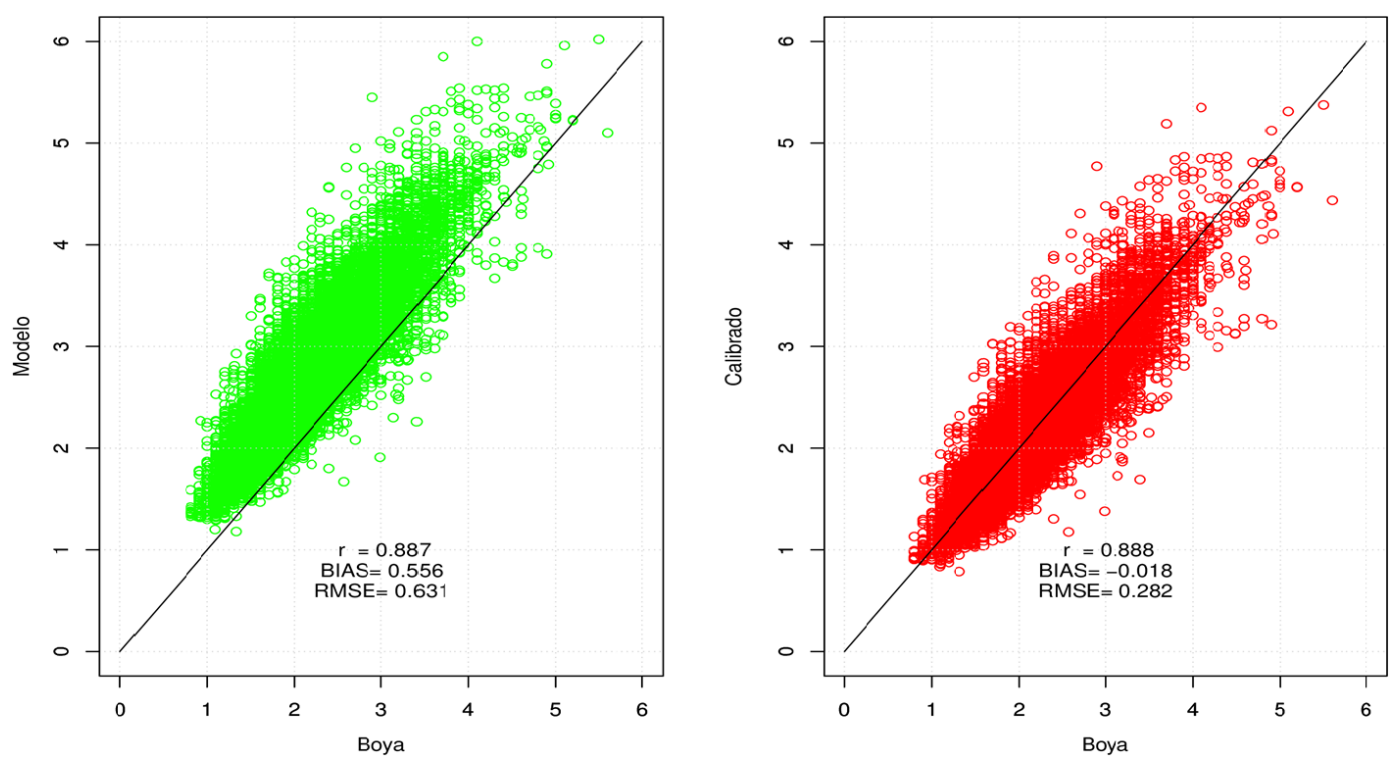

Figura 6: Correlación entre la información de reanálisis e instrumental de la boya 32012. Izquierda: sin calibrar. Derecha: calibrado. Fuente: propia. 

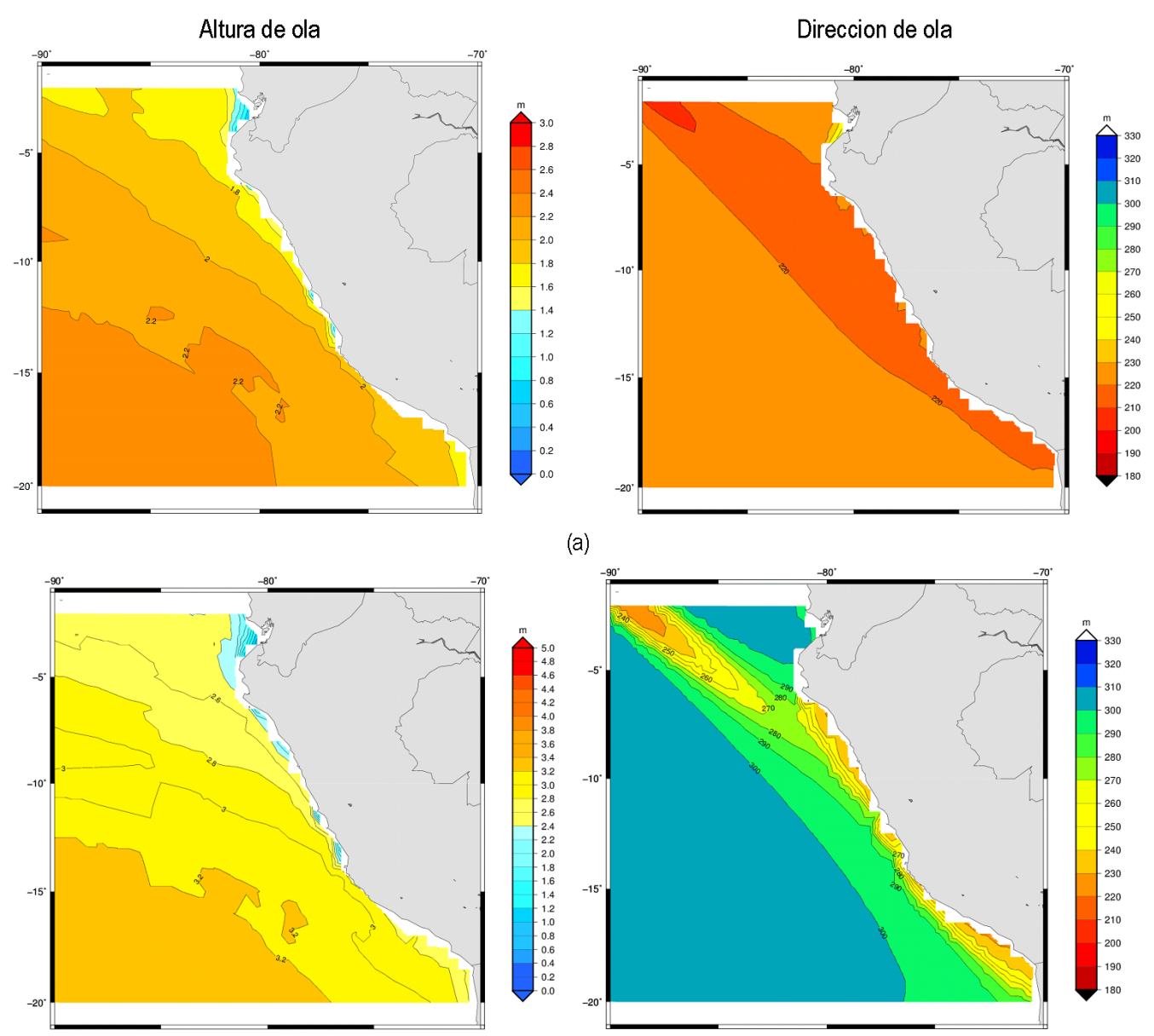

(a)
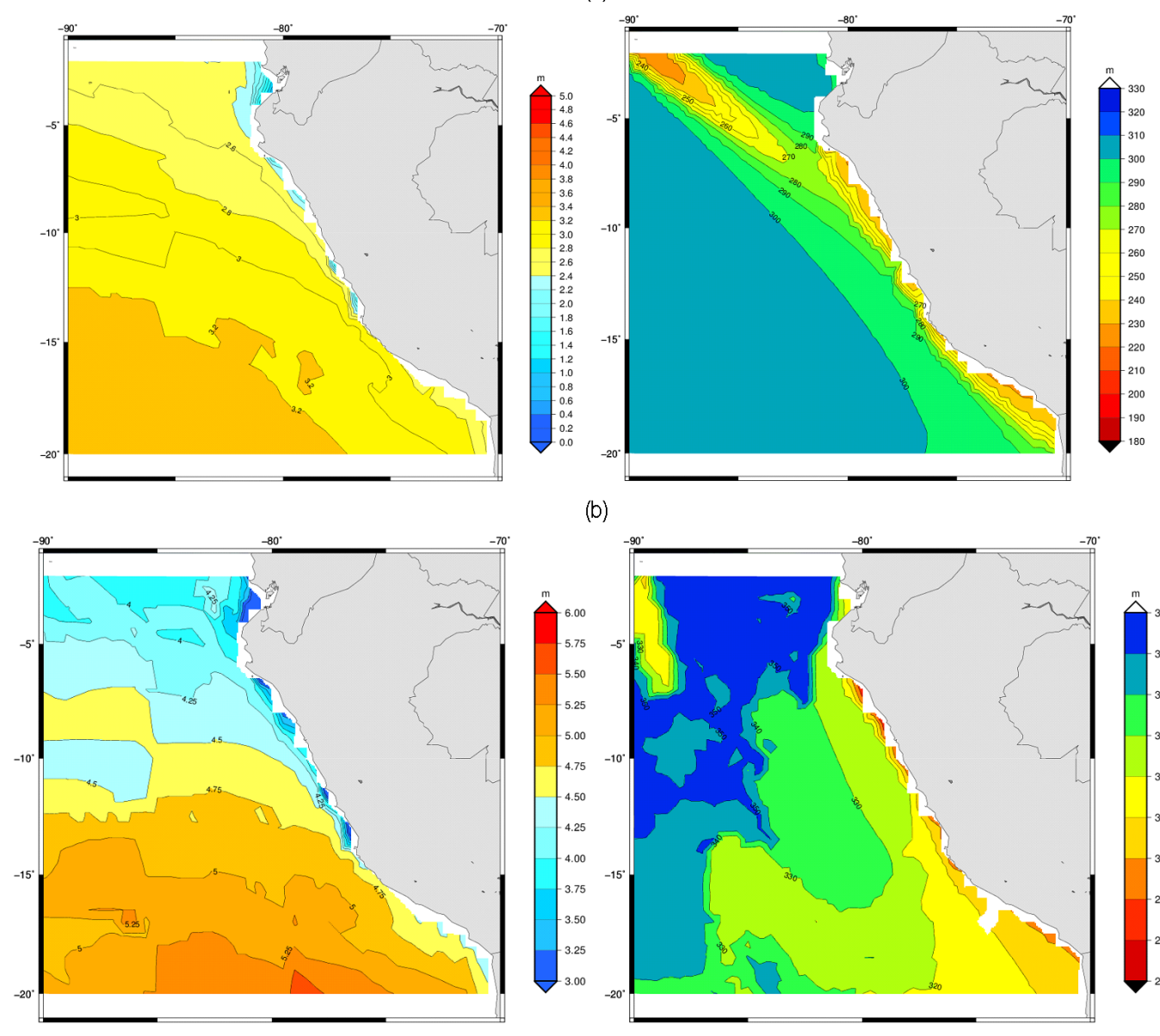

(b)

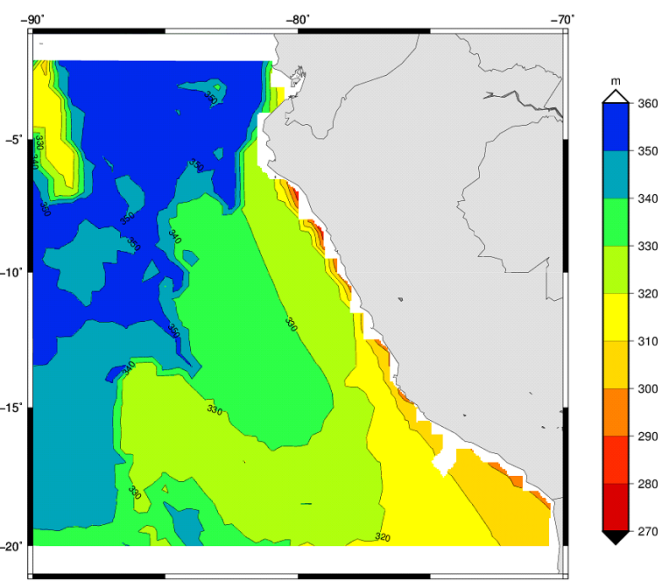

(c)

Figura 7: Distribución espacial de la altura significante y direccion pico. (a) Cuantil de 50\%, (b) Cuantil de $95 \%$ y (c) Máxima. Fuente: propia. 


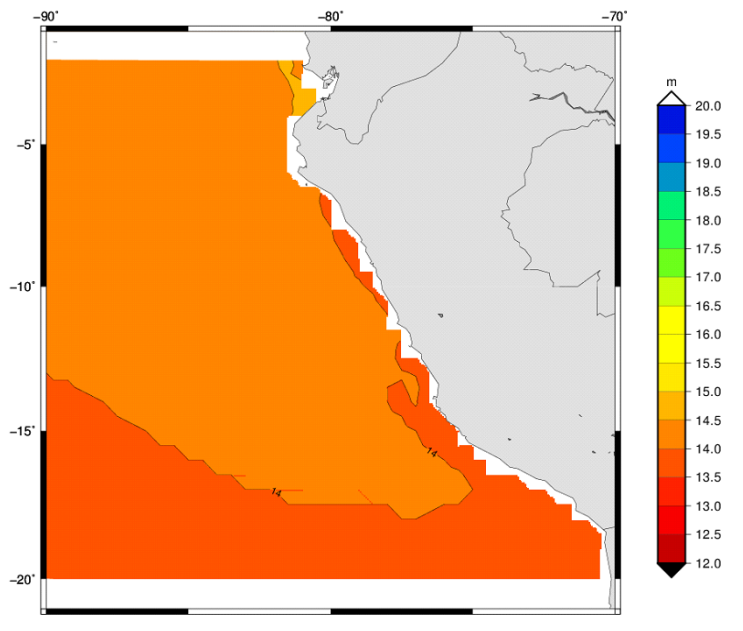

(a)

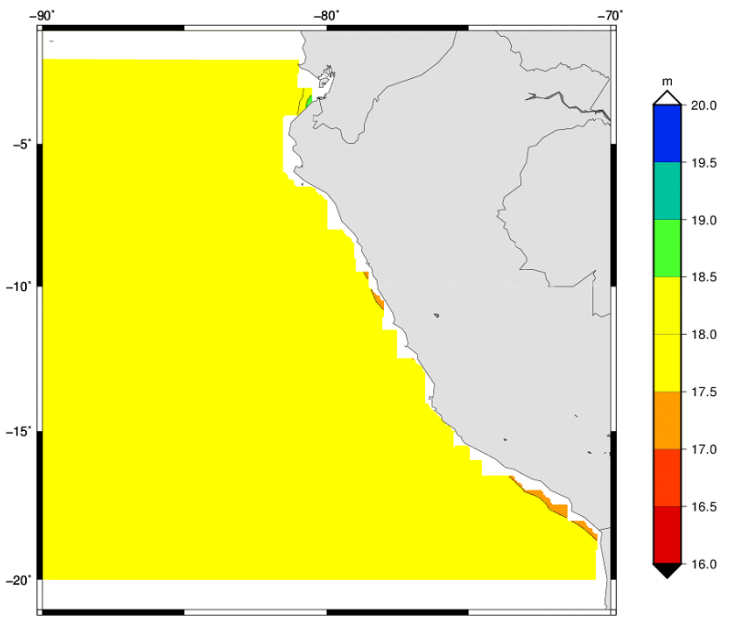

(b)

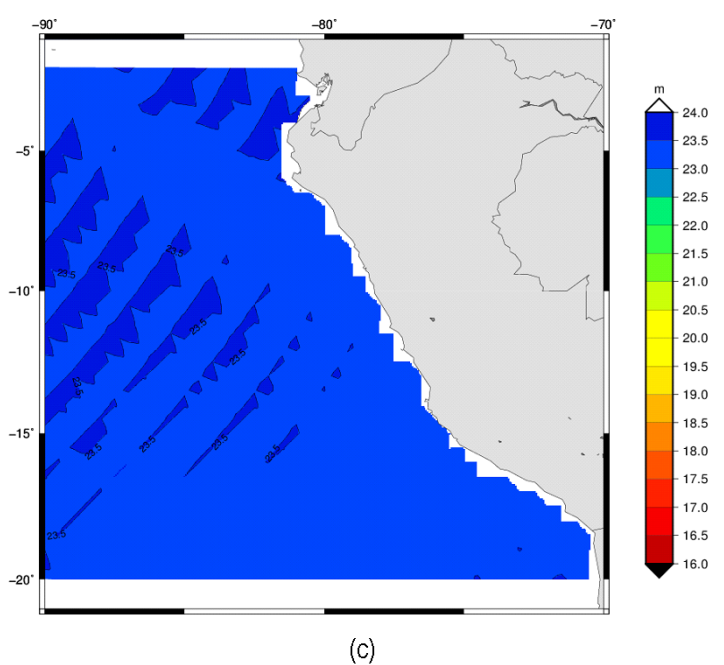

Figura 8: Distribución espacial del periodo pico. (a) Cuantil de $50 \%$, (b) Cuantil de $95 \%$ y (c) Máxima. Fuente: propia. 


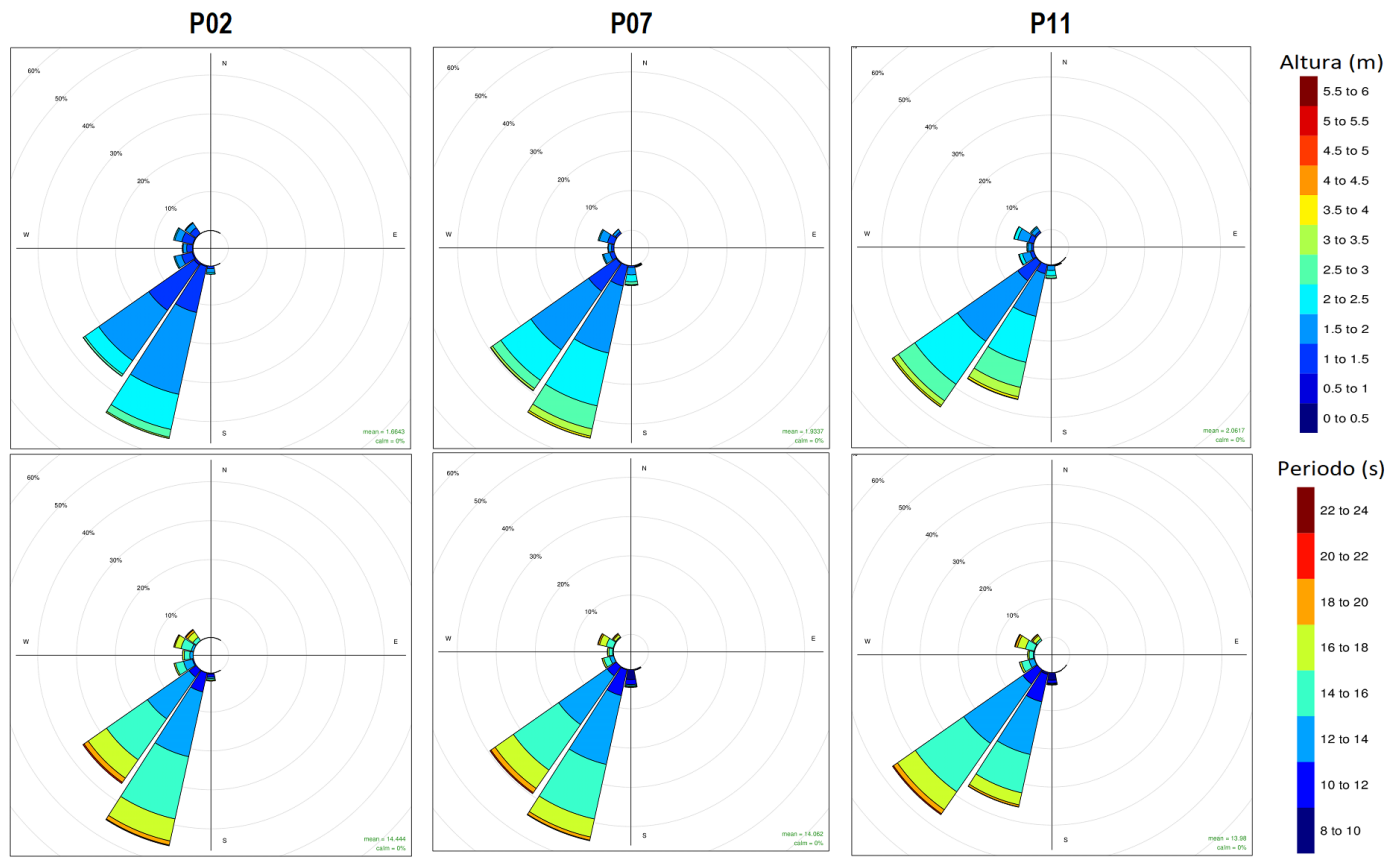

Figura 9: Rosa de altura y periodo de ola para los puntos P02, P07 y P11 seleccionados. Fuente: propia.
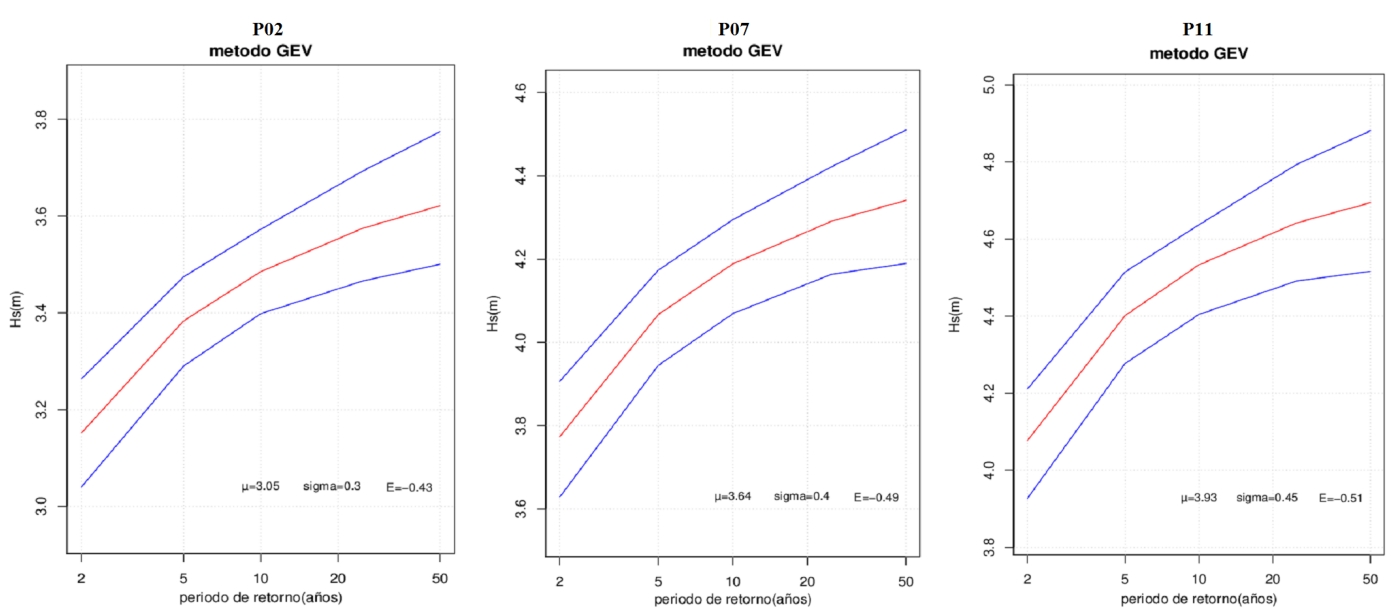

Figura 10: Rosa de altura y periodo de ola para los puntos P02, P07 y P11 seleccionados. Fuente: propia. 

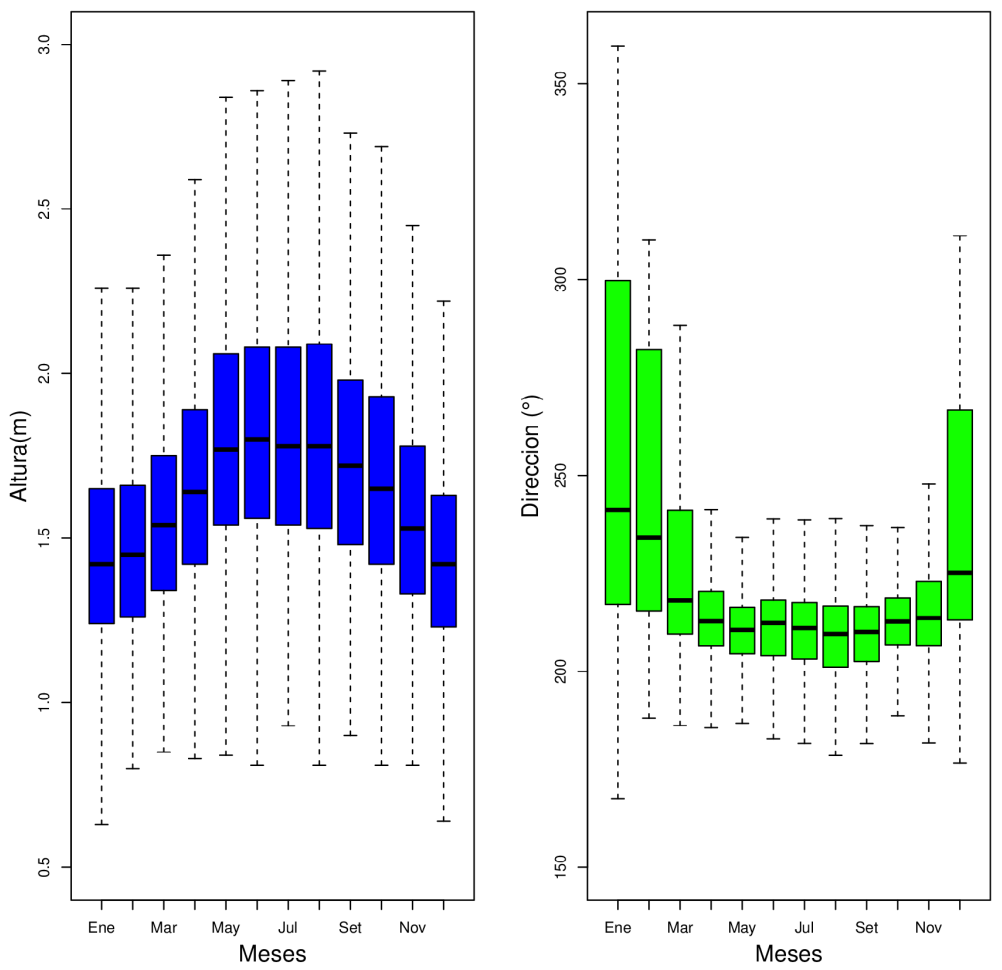

Figura 11: Variación estacional de altura y dirección de olas, para el punto P02.
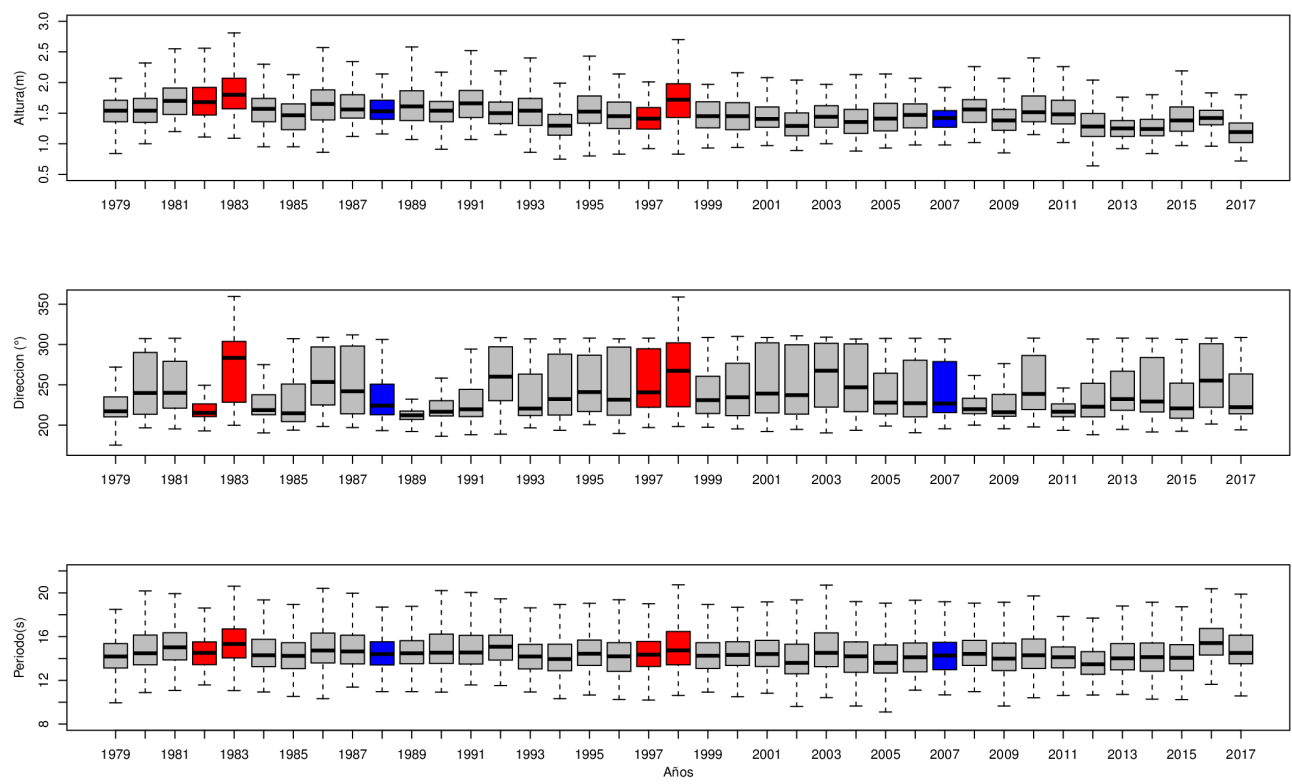

Figura 12: Variación anual de las olas para los meses de verano en el punto P02. Fuente: propia. 

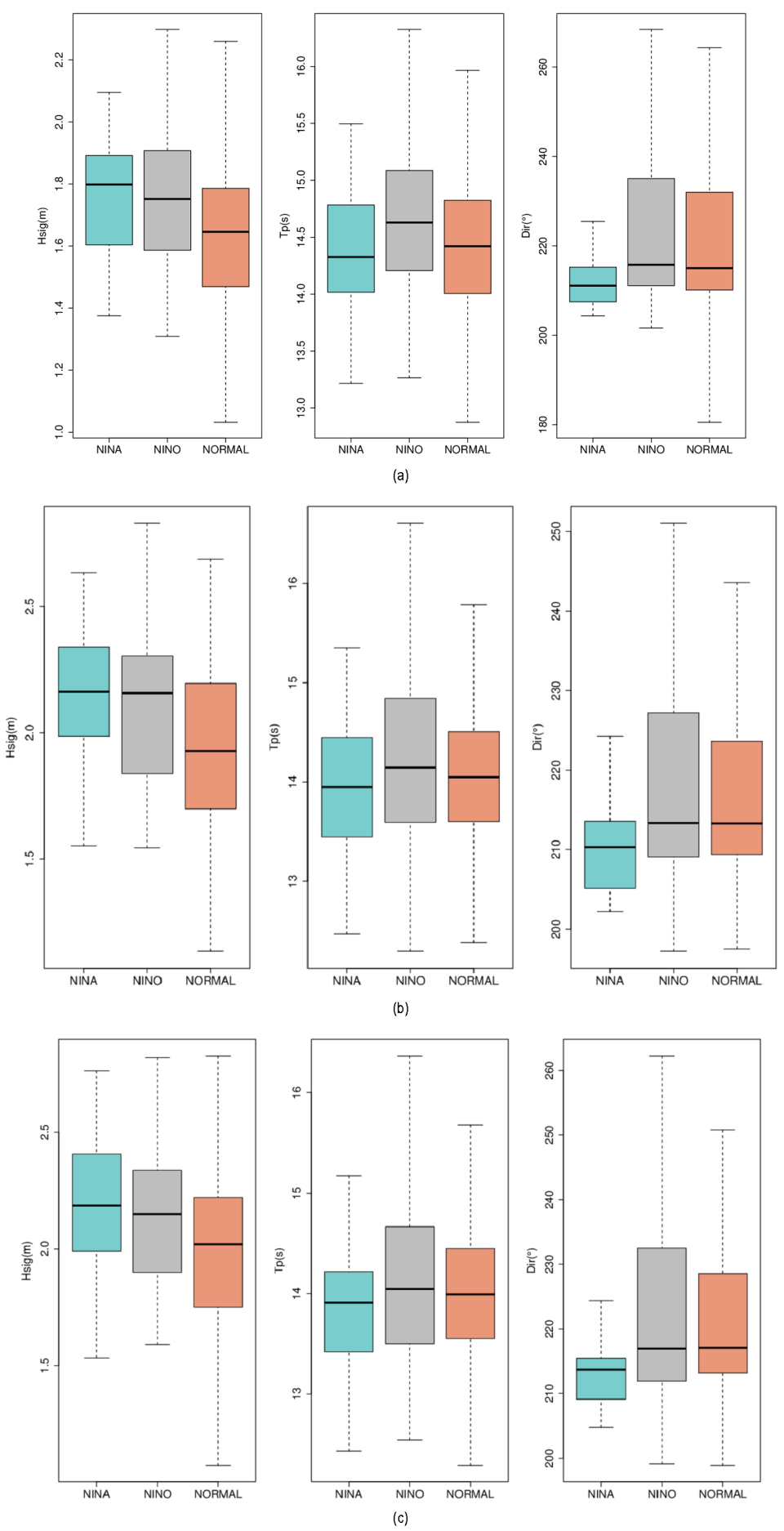

Figura 13: Distribución de altura significante, dirección y periodo en el punto P02 (a), P07 (b) y P11(c). Fuente: propia. 


\begin{tabular}{|l|c|c|c|c|c|c|c|c|c|c|}
\hline \multirow{2}{*}{ Punto } & \multirow{2}{*}{ Longitud } & \multirow{2}{*}{ Latitud } & \multicolumn{3}{|c|}{ Cuantiles (Altura de ola) } & \multicolumn{3}{c|}{ Cuantíles (Periodo de ola) } \\
\cline { 5 - 11 } & & $15 \%$ & $50 \%$ & $75 \%$ & $95 \%$ & $15 \%$ & $50 \%$ & $75 \%$ & $95 \%$ \\
\hline P01 & -84 & -2 & 1.42 & 1.65 & 1.93 & 2.43 & 13.2 & 14.3 & 15.6 & 17.8 \\
\hline P02 & -82 & -3 & 1.38 & 1.62 & 1.89 & 2.38 & 13.2 & 14.3 & 15.6 & 17.8 \\
\hline P03 & -82.5 & -5 & 1.45 & 1.69 & 1.98 & 2.50 & 12.9 & 14.2 & 15.5 & 17.8 \\
\hline P04 & -82 & -7 & 1.52 & 1.79 & 2.10 & 2.67 & 12.8 & 14.1 & 15.4 & 17.7 \\
\hline P05 & -80.5 & -8.5 & 1.46 & 1.72 & 2.06 & 2.66 & 12.8 & 14.1 & 15.4 & 17.7 \\
\hline P06 & -80 & -10 & 1.56 & 1.84 & 2.20 & 2.84 & 12.7 & 14.1 & 15.4 & 17.7 \\
\hline P07 & -79 & -11.5 & 1.58 & 1.86 & 2.22 & 2.86 & 12.7 & 14.1 & 15.4 & 17.7 \\
\hline P08 & -78 & -13 & 1.64 & 1.93 & 2.30 & 2.97 & 12.7 & 14.0 & 15.4 & 17.7 \\
\hline P09 & -78 & -15 & 1.70 & 2.01 & 2.39 & 3.13 & 12.7 & 14.0 & 15.4 & 17.7 \\
\hline P10 & -76 & -16.5 & 1.70 & 1.99 & 2.38 & 3.08 & 12.6 & 13.9 & 15.3 & 17.7 \\
\hline P11 & -76 & -18 & 1.68 & 1.99 & 2.36 & 3.04 & 12.6 & 13.9 & 15.3 & 17.7 \\
\hline P12 & -73 & -19 & 1.54 & 1.85 & 2.24 & 2.96 & 12.5 & 13.7 & 15.3 & 17.7 \\
\hline P13 & -88 & -2 & 1.46 & 1.70 & 1.99 & 2.50 & 12.8 & 14.2 & 15.4 & 17.7 \\
\hline P14 & -84 & -6 & 1.53 & 1.79 & 2.09 & 2.63 & 12.8 & 14.1 & 15.4 & 17.7 \\
\hline P15 & -80 & -14 & 1.75 & 2.06 & 2.43 & 3.14 & 12.7 & 14.1 & 15.4 & 17.8 \\
\hline P16 & -80 & -18 & 1.78 & 2.10 & 2.50 & 3.25 & 12.7 & 14.0 & 15.4 & 17.7 \\
\hline P17 & -88 & -6 & 1.67 & 1.94 & 2.25 & 2.80 & 13.0 & 14.3 & 15.5 & 17.8 \\
\hline P18 & -84 & -10 & 1.71 & 1.97 & 2.3 & 2.88 & 12.9 & 14.2 & 15.5 & 17.8 \\
\hline P19 & -84 & -14 & 1.78 & 2.09 & 2.46 & 3.15 & 12.8 & 14.2 & 15.5 & 17.8 \\
\hline P20 & -84 & -18 & 1.83 & 2.15 & 2.55 & 3.28 & 12.7 & 14.0 & 15.4 & 17.8 \\
\hline P21 & -88 & -10 & 1.74 & 2.02 & 2.36 & 2.99 & 12.9 & 14.3 & 15.5 & 17.8 \\
\hline P22 & -88 & -14 & 1.82 & 2.14 & 2.51 & 3.18 & 12.8 & 14.2 & 15.5 & 17.8 \\
\hline P23 & -88 & -18 & 1.87 & 2.19 & 2.59 & 3.34 & 12.7 & 14.1 & 15.4 & 17.8 \\
\hline
\end{tabular}

Tabla 5: Régimen medio de la altura y periodo de oleaje para cada punto de análisis Fuente: propia.

\begin{tabular}{|l|c|c|c|c|c|c|}
\hline \multirow{2}{*}{ Punto } & \multirow{2}{*}{ Método } & \multicolumn{5}{|c|}{ Periodo de retorno años de las alturas de olas $(\mathrm{m})$} \\
\cline { 3 - 7 } & & 2 & 5 & 10 & 20 & 50 \\
\hline \multirow{2}{*}{ P02 (Zona norte) } & GEV & 3.15 & 3.38 & 3.49 & 3.58 & 3.62 \\
\cline { 2 - 7 } & Gumbel & 3.10 & 3.44 & 3.66 & 3.95 & 4.16 \\
\hline \multirow{2}{*}{ P07 (Zona centro) } & GEV & 3.77 & 4.07 & 4.19 & 4.29 & 4.34 \\
\cline { 2 - 7 } & Gumbel & 3.70 & 4.21 & 4.55 & 4.98 & 5.30 \\
\hline \multirow{2}{*}{ P11 (Zona sur) } & GEV & 4.08 & 4.40 & 4.53 & 4.64 & 4.69 \\
\cline { 2 - 7 } & Gumbel & 3.98 & 4.57 & 4.96 & 5.44 & 5.81 \\
\hline
\end{tabular}

Tabla 6: Cálculo de la altura significante de ola para determinados periodos de retorno. Fuente : propia. 


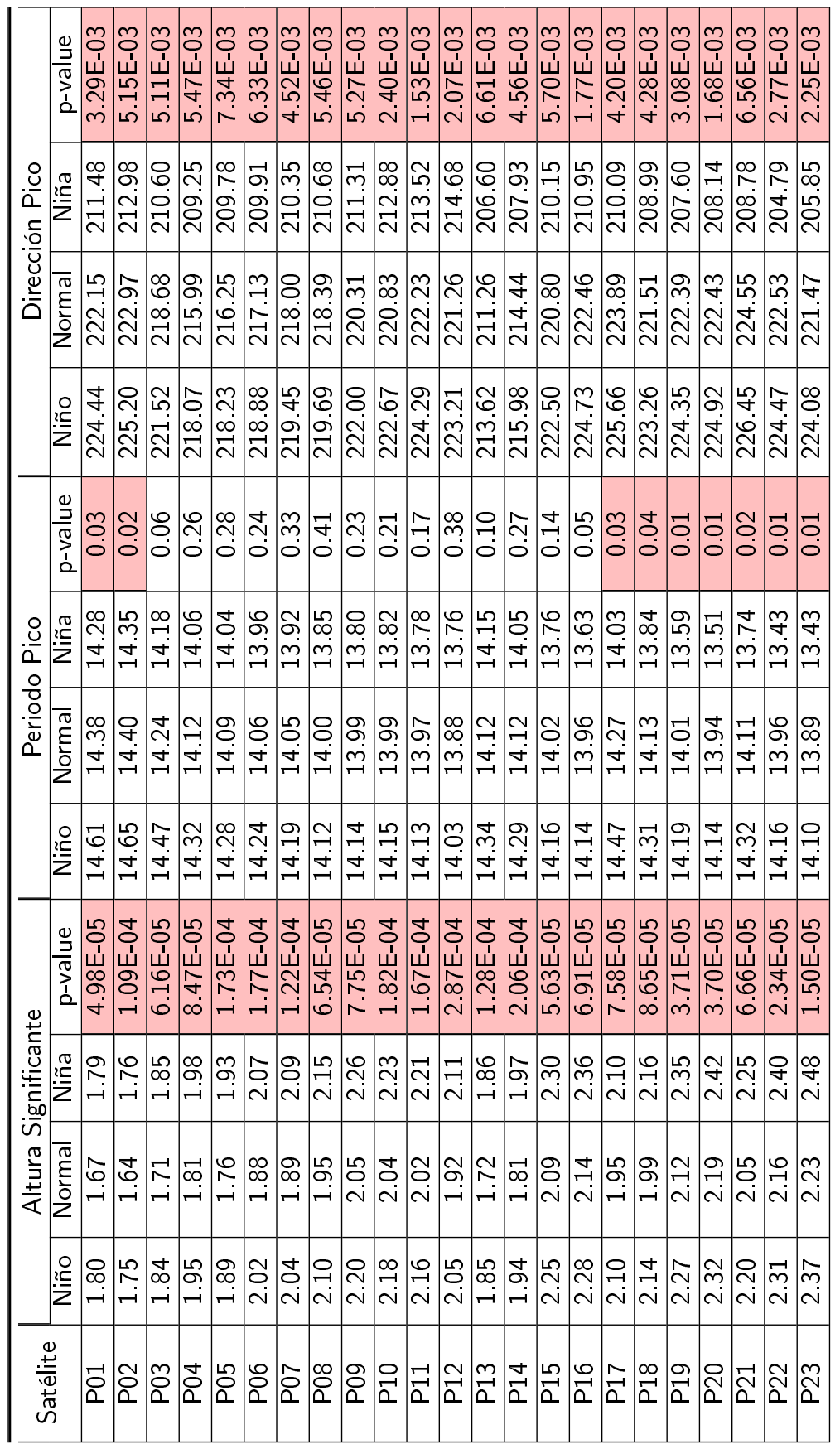

Tabla 7: Valores estadísticos de los valores promedios de olas durante eventos NIÑO, NIÑA Y NORMAL en los puntos de control seleccionados. Fuente: propia.

altura de olas entre 3.70 a $3.77 \mathrm{~m}$ mientras que, para 50 años, alturas máximas de retorno entre 4.34 a $5.30 \mathrm{~m}$.

- El punto P11 correspondiente a la zona sur del Pe- rú, muestra para 2 años retorno de olas entre 3.98 a $4.08 \mathrm{~m}$ mientras que, para 50 años, alturas máximas de retorno entre 4.69 a $5.81 \mathrm{~m}$.

Haciendo una comparación de las 2 distribuciones cal- 
culadas (Figura 11), se observa que en los primeros años los periodos de retorno de las alturas de olas presentan pocas variaciones en los 2 métodos, sin embargo, para los 50 años, el método Gumbel presenta mayores alturas de olas respecto al método GEV, por lo que se considera que el método de GEV presenta mejor ajuste de los datos, debido a que se encuentran muy próximos a los valores máximos reales encontrados en la serie de tiempo de 30 años, sin embargo no se descarta que los valores máximos calculados puedan darse en varias decenas de miles de años.

\section{Régimen Estacional}

Todos los puntos de olas calibrados muestran una tendencia estacional marcada observándose en la Figura 12 que entre los meses de Enero a Marzo (Verano) se presentan las menores alturas de olas con presencia de oleaje de Noroeste, mientras que entre julio a setiembre (Invierno) se presentan las mayores alturas de olas con dirección Sur-Suroeste.

\section{Fenómeno El Niño y La Niña}

Los análisis de las series temporales (Tabla 7) mostraron de manera general que los valores promedios de altura significante de olas se ven incrementadas durante la ocurrencia de El Niño y Niña, mientras que el periodo de olas sólo se ve incrementado durante El Niño, ya que durante periodos La Niña no se presentaron valores muy diferentes a los ocurridos en años normales. Por otro lado, la dirección promedio del oleaje durante El Niño se ve incrementado de manera ligera, y sucede lo contrario durante La Niña, aunque en los tres eventos el oleaje siguió siendo del Suroeste. Por otro lado, los gráficos de distribución mostrados en la Figura 14, guarda relación con lo mostrado en la Tabla 7 , siendo lo más resaltante que durante El Niño el oleaje para el cuantil 75 presenta una ocurrencia predominante del Oeste, mientras que en ocurrencia de La Niña, prácticamente todo el oleaje proviene de la dirección Suroeste. Es conocido que el evento El Niño cambia las condiciones climáticas en verano (enero a marzo), por lo que se eligió estos meses para evaluar las variaciones anuales de las olas (Figura 13), los cuales evidencian que durante los años EI Niño 1982-1983 y 1997-1998 se muestra un incremento en las alturas de olas, con presencia de oleaje del Noroeste y periodos en el orden de 16 segundos; sin embargo, durante eventos La Niña no se observa cambios considerables respecto a otros años para los meses de verano para el punto de olas seleccionado (P02).

\section{Conclusiones}

Se realizó la calibración de los datos del reanálisis numérico con información instrumental proveniente de satélite y boyas cercanas a la zona de estudio. Obteniéndose una serie de tiempo de olas de 1979 a 2017 con una correlación aceptable $(0.729<\mathrm{r}<0.933)$ en prácticamente todo el dominio estudiado. El oleaje en los puntos representativos de la zona norte centro y sur del Perú, presentan dirección predominante del Sur-suroeste en la zona norte y centro, mientras que, en la zona sur se presenta dirección predominante del Suroeste.

El régimen medio de oleaje muestra de manera general que la zona norte del país presenta menores alturas de olas, aproximadamente $1.62 \mathrm{~m}$ (P02), mientras que la zona centro y sur, muestra alturas de $1.86 \mathrm{~m}$ y $1.99 \mathrm{~m}$ para los puntos P07 y P11 respectivamente. Por otro lado, el régimen extremal muestra la probabilidad que ocurra un temporal en aproximadamente 50 años, de alturas de olas entre 3.6 y $4.1 \mathrm{~m}$ en la zona norte del país, mientras que en la zona sur se esperaría un temporal con alturas entre 4.6 a $5.8 \mathrm{~m}$. El régimen estacional nos indica que en el invierno se presentan las mayores alturas de oleaje y que provienen de la dirección suroeste, sin embargo, en verano existe mayor incidencia de oleajes de dirección Noroeste y con alturas de olas de $1.5 \mathrm{~m}$.

Los resultados de la influencia de fenómenos como $\mathrm{El}$ Niño y La Niña muestran que el oleaje se ve alterado con respecto a la ocurrencia de años normales, esto fue evidenciado en todos los puntos de análisis, donde se observó que las alturas de oleaje se vieron incrementadas durante El Niño y La Niña. Para el periodo pico de oleaje, la variación con respecto a años normales es muy ligera. En cuanto a la dirección se observa que cuando se presenta La Niña el oleaje presenta dirección predominante del Suroeste (observado, en todos los puntos de análisis); sin embargo, cuando hay la presencia de EI Niño la dirección de oleaje presenta mayor variabilidad, observándose presencia de olas que provienen del oeste y noroeste.

\section{Referencias}

[1] B.Dewitte,S. Illig, L. Renault, K. Goubanova, K. Takahashi (2011). Modes of covariability between sea surface temperature and wind stress intraseasonal anomalies along the coast of Peru from satellite observations (2000-2008). Journal of Geophysical Research, 116(C4).

[2] Beyá M., J., Gallardo, A., Álvarez, M., Hidalgo, H., \& Valdivia, J. (2016). Atlas de Oleaje de Chile. Primera edición. Obtenido de Escuela de Ingeniería Civil Oceánica - Universidad de Valparaíso: http://www. oleaje.uv.cl/descargas.html

[3] CENEPRED (2012). Escenarios de Riesgos ante la Probabilidad de Ocurrencia del Fenómeno El Niño. Lima.

[4] Chawla, A. T.-H.-M. (2013). A multi grid wave fore- 
casting model: A new paradigm in operational wave forecasting. Weather and Forecasting.

[5] ENFEN (2012). Definición Operacional de los Eventos El Niño y La Niña y sus Magnitudes en la Costa del Perú. Lima.

[6] Environmental Modeling Center. (s.f.). Obtenido de ftp://polar.ncep.noaa.gov/pub/history/ waves/multi_1/estado P. d. (s.f.). Clima medio de oleaje.

[7] Farquhar, C. R., Deinghton, H., Busswell, G., Snaith, H. M., Ash, E., Collard, F., . . . Pinnock, S. (2013). GLOBWAVE: A GLOBAL WAVE DATA PORTAL.

[8] Francois-Thomas, Y., Ricaurte-Villota, C., \& CocaDomínguez, O. (2016). Atlas climatológico del océano Pacífico colombiano y áreas adyacentes. Obtenido de INVEMAR: http://cinto.invemar. org.co/alfresco/d/d/workspace/SpacesStore/ 525648f3-b84d-4a0a-b4f8-cc394b1054bc/ATLAS_ CLIMATOLOGICO_Pacifico.pdf

[9] Ge Chen, Bertrand Chapron, Robert Ezraty, \& Douglas Vandemark. (2002). A Global View of Swell and Wind Sea Climate in the Ocean by Satellite Altimeter and Scatterometer. American Meteorological Society, 1849-1859.

[10] GlobWave. (2013). Obtenido de http://globwave. ifremer.fr/

[11] Gregory C. Johnson and Michael J. McPhaden , G. Daft Rowe, \& McTaggart , K. (2002). Upper equatorial Pacific Ocean current and salinity variability during the 1996-1998 El Niño- La Niña Cycle. Journal of Geophysical Research, 1037-1053.

[12] Hemer, M. A. (2010). Variability and trends in the directional wave climate of the Southern Hemisphere. International Journal of Climatology, 475-491.

[13] IHCantabria. (2014). AMEVA-Manual de Usuario v1.4.1. Obtenido de IHCantabria: http://ihameva. ihcantabria.com/

[14] Klaus Wyrtki. (1977). Sea Level during the 1972 El Niño . American Meteorological Society, 779-787.

[15] Krogstad, H. E., \& Barstow, S. F. (1999). Satellite wave measurement for coastal engineering application. Coastal Engineering Vol 37, 283-307.
[16] Lavado-Casimiro, W., \& Espinoza, J. (2014). Impactos de El Niño y La Niña en las lluvias del Perú (19652007). Revista Brasileira de Meteorologia, 171-182.

[17] Macafee , A., \& Wong, S. (2007). Extreme Value Analysis of Tropical Cyclone Trapped-Fetch Waves. Journal of Applied Meteorology and Climatology, 1501-1522.

[18] Mark A. Hemer, Yalin Fan, Nobuhito Mori,Alvaro Sea. (Mayo 2013). Projected changes in wave climate from a multi-model ensemble. Nature Climate Change, 471-476.

[19] Mei Zhao, Harry H. Hendon, Yonghong Yin, Oscar. (2016). Variations of Upper-Ocean Salinity Associated with ENSO from PEODAS Reanalyses. Journal of Ocean University of China, 2077-2094.

[20] Minguez, R., Espejo, A., Tomas, A., Mendez, F., \& Losada, I. (2011). Directional Calibration of Wave Reanalysis Databases Using Instrumental Data. Journal of Atmospheric and Oceanic Technology.

[21] NOAA. (2017). National Data Buoy Center. Obtenido de http://www.ndbc.noaa.gov/

[22] NOAA. (s.f.). Marine Modeling and Analysis Branch. Obtenido de http://polar.ncep.noaa.gov/waves/ hindcasts/nopp-phase1.php

[23] Reguero, B. G., Menéndez, M., Minguez, R., \& Losada, I. J. (2012). A Global Ocean Wave (GOW) calibrated reanalysis from 1948 onwards. Coastal Engineering, 38-55.

[24] Scott A. Stephens, \& D.L. Ramsay. (2014). Extreme cyclone wave climate in the Southwest Pacific Ocean: Influence of the EI Niño Southern Oscillation and projected climate change. Global and Planetary Change, 13-26.

[25] Tolman, H.L., B. Balasubramaniyan, L.D. Burroughs,. (2002). Development and implementation of wind generated ocean surface wave models at NCEP. Weather and Forecasting, 311-333.

[26] Vera San Martín, L., \& Marín Villalta, S. (2015). Acta Oceanografica del Pacifico. (págs. 45-56). Guayaquil-Ecuador. 